\title{
Ability of Different Measures of Adiposity to Identify High Metabolic Risk in Adolescents
}

\author{
Carla Moreira, ${ }^{1}$ Rute Santos, ${ }^{1}$ Susana Vale, ${ }^{1}$ Paula C. Santos, ${ }^{1,2}$ Sandra Abreu, ${ }^{1}$ \\ Ana I. Marques, ${ }^{1}$ Luísa Soares-Miranda, ${ }^{1}$ and Jorge Mota $^{1}$ \\ ${ }^{1}$ Research Centre in Physical Activity, Health and Leisure, Faculty of Sport, University of Porto, Rua Dr. Plácido Costa 91, \\ 4200-450 Porto, Portugal \\ ${ }^{2}$ Department of Physiotherapy, School of Health Technology of Porto, IPP, Portugal
}

Correspondence should be addressed to Carla Moreira, carla_m_moreira@sapo.pt

Received 21 March 2011; Revised 13 May 2011; Accepted 16 May 2011

Academic Editor: Gianluca Iacobellis

Copyright ( $\odot 2011$ Carla Moreira et al. This is an open access article distributed under the Creative Commons Attribution License, which permits unrestricted use, distribution, and reproduction in any medium, provided the original work is properly cited.

\begin{abstract}
Introduction. This study aimed to evaluate the screening performance of different measures of adiposity: body mass index (BMI), waist circumference (WC), and waist-to-height ratio (WHtR) for high metabolic risk in a sample of adolescents. Methods. A crosssectional school-based study was conducted on 517 adolescents aged 15-18, from the Azorean Islands, Portugal. We measured fasting glucose, insulin, total cholesterol (TC), HDL-cholesterol, triglycerides, and systolic blood pressure. HOMA and TC/HDL$\mathrm{C}$ ratio were calculated. For each of these variables, a Z-score was computed by age and sex. A metabolic risk score (MRS) was constructed by summing the Z-scores of all individual risk factors. High risk was considered when the individual had $\geq 1 \mathrm{SD}$ of this score. Receiver-operating characteristics (ROC) were used. Results. Linear regression analyses showed that, after adjusting for age and pubertal stage, all different measures of adiposity are positively and significantly associated with MRS in both sexes, with exception of WHtR for boys. BMI, WC, and WHtR performed well in detecting high MRS, indicated by areas under the curve (AUC), with slightly greater AUC for BMI than for WC and WHtR in both sexes. Conclusion. All measures of adiposity were significantly associated with metabolic risk factors in a sample of Portuguese adolescents.
\end{abstract}

\section{Introduction}

The prevalence of overweight and obesity is high in most parts of the world, and this is particularly alarming not only for the increasing risk of multiple comorbidities [1], but also due to the tendency of childhood overweight and obesity to track into adulthood [2].

The metabolic syndrome (MetS), a cluster of several cardiovascular disease risk factors, is a complex entity of metabolic disorders that significantly increases the risk of type II diabetes and cardiovascular disease beyond that of its individual components [3]. The emergence of MetS parallels the rising rates of overweight and obesity observed in youth worldwide [4].

A variety of anthropometric indices have been used as a proxy for total and abdominal fat to assess risk for diseases, particularly cardiovascular diseases (CVD) and diabetes [5]. Epidemiologic studies have shown that body mass index (BMI), the most widely recognised measure of obesity, is a powerful predictor of CVD [6]. Nevertheless, waist circumference (WC) and waist-to-height ratio (WHtR) measures of central obesity have yielded important insight as well and also provide information on the risk of CVD [7].

Although different anthropometrical measures of obesity have been proposed, it remains unclear which measures of adiposity best predict the role of metabolic risk factors. The aim of this study was to determine the ability of different measures of adiposity, namely, BMI, WC, and WHtR to discriminate between low/high metabolic risk using receiveroperating characteristic (ROC) curves in a sample of Portuguese adolescents from the Azorean Archipelago.

\section{Methods}

2.1. Study Design and Sampling. Data for the present study derived from a longitudinal school-based study, the Azorean 
Physical Activity and Health Study II, aimed to evaluate physical activity, physical fitness, overweight/obesity prevalence, health-related quality of life, and related factors. Details on the study design and sampling strategy are reported elsewhere [8]. The final sample included in this crosssectional analysis was comprised of 517 adolescents (297 girls and 220 boys) aged 15 to 18 .

All participants in this study were informed about the objectives of the work, and the parent or guardian of each participant provided written informed consent. The study was approved by the faculty and the Portuguese Foundation for Science and Technology ethics committee and conducted in accordance with the Declaration of Helsinki for Human Studies of the World Medical Association.

2.2. Anthropometric Measures. Height was measured to the nearest millimeter in bare or stocking feet with the adolescent standing upright against a stadiometer (Holtain Ltd., Crymmych, Pembrokeshire, UK). Weight was measured to the nearest $0.10 \mathrm{~kg}$, with adolescents lightly dressed using a portable electronic weight scale (Tanita Inner Scan BC 532). BMI was calculated as body weight $(\mathrm{kg})$ divided by body height $\left(\mathrm{m}^{2}\right)$. WC measurements were taken as described by Lohman et al. [9]. The waist and height were used to compute the WHtR.

2.3. Pubertal Stage. To determine the pubertal stage (ranging from stage 1 to 5), each subject was asked to self-assess his/her stage of secondary sex characteristics. Stages of breast development in girls and genital development in boys were evaluated according to the criteria of Tanner and Whitehouse [10].

2.4. Blood Sampling. Blood samples were collected from the antecubital vein between 8:00 and 10:00 a.m., in a sitting position after ten hours of fasting. Blood samples were drawn in vacuum tubes gel (Sarstedt) in order to obtain values of plasmatic total cholesterol (TC), high-density lipoprotein cholesterol (HDL-C), triglycerides (TG), glucose, and insulin. The following analyses were measured on a Cobas Integra 400 Plus (ROCHE Diagnostics, Indianapolis, Ind, USA): TC, HDL-cholesterol, TG, and glucose. The fasting serum insulin was measured on an Immulite 2000, (Diagnostic Products Corporation, Los Angeles, Calif). The ratio of total cholesterol to HDL-C was calculated as an index of atherogenic lipid profile [11]. The homeostatic model assessment (HOMA), calculated as the product of basal glucose and insulin levels divided by 22.5 , was used as a proxy measure of insulin resistance [12]. The biochemical evaluation of all participants from the different islands was conducted in the same laboratory.

2.5. Blood Pressure. Blood pressure (BP) was measured using the Dynamap adult/pediatric vital signs monitors, model BP 8800 (Critikon, Inc., Tampa, Fla, USA). Measurements were taken by trained nurses, and with all adolescents were required to sit and rest for at least five minutes prior to the BP test. The participants were in a seated, relaxed position with their feet resting flat on the ground. Two measurements in the right arm were taken after five and ten minutes of rest. The mean of these two measurements was considered. If the two measurements differed by $2 \mathrm{mmHg}$ or more, a third measure was taken.

2.6. Metabolic Risk Score. Since there is no consensus regarding the establishment of a universal criterion for definition of the MetS in adolescents, we decided to compute a continuous metabolic risk score (MRS) from the following measurements: TC/HDL-C ratio, TG, HOMA, and systolic BP. For each of these variables, a Z-score was computed by age and sex. Then, an MRS was constructed by summing the Z-scores of all individual risk factors. High risk was considered when the individual had $\geq 1 \mathrm{SD}$ of this score. The score only applies to this study population. A similar Z-score approach has been used previously in children and adolescents [13].

2.7. Statistical Analysis. Comparisons between groups involved Student $t$-test for continuous variables. Linear regression analyses were used to study the relationship between different measures of adiposity and low and high MRS, adjusting for age and pubertal stage. Receiveroperating characteristic (ROC) curve analyses were used to analyse the potential diagnostic accuracy of the different measures of adiposity to discriminate between low and high MRS. The area under the curve (AUC) and 95\% confidence interval (CI) were calculated. The AUC represents the ability of the test to correctly classify adolescents having a low and high MRS. The values of AUC range between 1 (perfect test) and 0.5 (worthless test). Data were analyzed using the PASW Statistic v.18 (SPSS, Chicago, Ill, USA) and Med Calc software v.10.4.5 (MedCalc Software, Mariakerke, Belgium). A $P$ value under 0.05 denoted statistical significance.

\section{Results}

Descriptive characteristics of the adolescents are shown in Table 1. Girls had lower height, weight, WC, systolic BP, glucose, and TC/HDL-C levels and higher TC and HDL-C than boys $(P<0.05$ for all). All adolescents reported to be in Tanner stage 4 or 5 .

Linear regression analyses, adjusted for age and pubertal stage, showed that BMI (girls: $\beta=0.291,95 \%$ CI: $0.219-$ 0.364, $P<0.001$; boys: $\beta=0.396$, 95\% CI: $0.324-0.468$, $P<0.001$ ), WC (girls: $\beta=0.086,95 \%$ CI: $0.060-0.111$, $P<0.001$; boys: $\beta=0.121,95 \%$ CI: $0.093-0.150, P<0.001$ ), and WHtR (girls: $\beta=1.738,95 \%$ CI: $0.555-2.922, P=$ 0.004; boys: $\beta=1.220,95 \%$ CI: $-0.209-2.648, P=0.09$ ) were positively and significantly associated with MRS in both sexes, with exception of WHtR for boys.

ROC curve analysis showed that all measures of adiposity performed well on average in identifying high MRS, as indicated by AUC greater than 0.7. The ROC performance of BMI showed a better discriminatory accuracy than WC and WHtR in predicting high MRS in both sexes. In boys, the ROC performance of BMI was slightly better than in 
TABLE 1: Descriptive characteristics of the study sample.

\begin{tabular}{lccc}
\hline Variables & Total $(n=517)$ & Girls $(n=297)$ & Boys $(n=220)$ \\
\hline Age, years & $16.5 \pm 0.9$ & $16.5 \pm 1.0$ & $16.4 \pm 0.8$ \\
Height, cm & $165.0 \pm 13.6$ & $160.0 \pm 11.0$ & $170.2 \pm 1.0^{*}$ \\
Weight, kg & $63.1 \pm 12.5$ & $58.6 \pm 10.0$ & $69.3 \pm 13.1^{*}$ \\
BMI, kg/m ${ }^{2}$ & $22.9 \pm 3.7$ & $22.7 \pm 3.5$ & $22.7 \pm 3.5$ \\
Waist circumference, cm & $79.3 \pm 10.7$ & $78.3 \pm 10.3$ & $80.7 \pm 11.1^{*}$ \\
Systolic BP, mmHg & $115.2 \pm 15.3$ & $111.8 \pm 13.8$ & $120.0 \pm 16.1^{*}$ \\
Diastolic BP, mmHg & $66.4 \pm 9.4$ & $66.3 \pm 10.0$ & $66.5 \pm 8.6$ \\
Total cholesterol, mg/dL & $161.9 \pm 32.3$ & $169.3 \pm 33.6$ & $151.8 \pm 27.5^{*}$ \\
HDL-cholesterol, mg/dL & $55.6 \pm 13.4$ & $59.3 \pm 13.0$ & $50.5 \pm 12.3^{*}$ \\
Triglycerides, mg/dL & $70.7 \pm 35.1$ & $72.5 \pm 34.6$ & $68.1 \pm 35.6$ \\
Total cholesterol/HDL-C ratio & $3.0 \pm 0.7$ & $2.9 \pm 0.8$ & $3.1 \pm 0.7^{*}$ \\
Fasting glucose, $(\mathrm{mg} / \mathrm{dL})$ & $86.8 \pm 9.2$ & $84.6 \pm 8.7$ & $89.7 \pm 9.1^{*}$ \\
Fasting insulin, $(\mathrm{uU} / \mathrm{mL})$ & $9.1 \pm 6.0$ & $9.3 \pm 5.1$ & $8.7 \pm 7.0$ \\
HOMA & $1.9 \pm 1.3$ & $1.9 \pm 1.1$ & $1.9 \pm 1.6$ \\
Metabolic risk score & $0.1 \pm 3.1$ & $0 \pm 3.1$ & $0.1 \pm 3.2$ \\
\hline
\end{tabular}

Data are means \pm standard deviations. ${ }^{*} P<0.05$ for sex comparisons (one-tailed $t$-test). BMI, body mass index; BP, blood pressure; HDL, high-density lipoprotein; ${ }^{a}$ obtained by summing individual risk factors (total cholesterol/HDL-C ratio, triglycerides, HOMA, and systolic blood pressure) age- and sexstandardized scores.

girls. The AUC of BMI were significantly different from WC $(P<0.05)$ for the whole sample. In girls, the AUC of WC was significantly different from WHtR $(P<0.05)$.

A BMI of $23.7 \mathrm{~kg} / \mathrm{m}^{2}$ for girls and $27.0 \mathrm{~kg} / \mathrm{m}^{2}$ for boys, a WC of $83 \mathrm{~cm}$ for girls and $92 \mathrm{~cm}$ for boys, and a WHtR of 0.55 for girls and 0.49 for boys were found to be optimal cutoffs for defining high MRS in this adolescent population (Table 2).

\section{Discussion}

The main findings of this study suggested that BMI provides a marginally superior tool for discriminating high MRS compared with WC and WHtR, for both sexes. Slightly higher pooled AUC were observed in boys compared to girls, suggesting that discrimination is more precise, on average, in male. Linear regression analyses showed that all different measures of adiposity were positively and significantly associated with MRS in both sexes, with the exception of WHtR for boys.

Jung et al. showed that BMI had the best predictive power to identify metabolic syndrome, its components, and markers for low-grade inflammation [14], which is in agreement with our results. The BMI and WC are widely used to define overweight and obesity across populations [15]. The BMI has been used to predict body composition and health risk [16], whereas WC indicates visceral adipose tissue and can predict health risks in children [17]. Both BMI and WC are simple measures to use and interpret, yet they have some limitations. The BMI does not distinguish fat mass from fat-free mass or between different body fat distributions [18], and for WC, there are currently no agreements about a health-related classification for children and adolescents. Several previous studies considered the 90th percentile as a cut-off point for high WC, whereas other studies consider the 75th or 70th percentile as a cutoff point. Both of these measures are also age and-sex dependent.

The WHtR has been significantly associated with cardiovascular risk factors, due to abdominal obesity both in adults and children [19]. The higher AUC value for WHtR than for WC could be due to the fact that WHtR takes into account differences in body height. Contrary to our findings, some studies have shown that WHtR is better for classifying obesity related to cardiovascular risk than BMI and WC $[5,20]$. In girls, the AUC value for WHtR is slightly better than AUC value for WC. Despite the AUC value for BMI was slightly higher than the other two measures in girls, the AUC value for WHtR showed higher sensibility than AUC value for BMI.

There is no agreement for which anthropometrical measures of adiposity best predict a role of unfavorable cardiovascular risk factors. Studies carried out among children and adolescents in Cyprus [21] and Japan [5] concluded that both WC and WHtR are better predictors of TC, TG, HDL-cholesterol, low-density lipoprotein cholesterol, and systolic and diastolic BP levels than BMI. Also, the Bogalusa study reported that WC and WHR were related to adverse levels of TG and HDL-cholesterol, independently of race, sex, age, weight, and height [22]. Conversely, BMI is the measure of obesity most used and has been shown to be extremely effective when used in longitudinal studies [7].

Nevertheless, we were unable to draw cause-effect conclusions and to make observations over the time because of the cross-sectional nature of our data and the multifactorial etiology of high MRS. However, the association between these measures of adiposity and the clustering of metabolic risk factors has not been studied in this population, making it one of the strengths of this 
TABLE 2: Cut-off values, sensitivity, and specificity for the association of different measures of adiposity with metabolic risk score by sex.

\begin{tabular}{|c|c|c|c|}
\hline & All & Girls & Boys \\
\hline \multicolumn{4}{|l|}{ BMI } \\
\hline BMI cut-off $\left(\mathrm{kg} / \mathrm{m}^{2}\right)$ & $>23.6$ & $>23.7$ & $>27.0$ \\
\hline Sensitivity (\%) & 79.4 & 76.3 & 66.7 \\
\hline Specificity (\%) & 72.8 & 71.8 & 94.7 \\
\hline \multirow{2}{*}{ AUC } & $0.807(0.770-0.840)$ & $0.772(0.720-0.818)$ & $0.852(0.798-0.896)$ \\
\hline & $P<0.001^{\ddagger}$ & $P<0.001$ & $P<0.001$ \\
\hline \multicolumn{4}{|l|}{ WC } \\
\hline WC cut-off $(\mathrm{cm})$ & $>84$ & $>83$ & $>92$ \\
\hline Sensitivity (\%) & 66.2 & 65.8 & 60.0 \\
\hline Specificity (\%) & 79.7 & 75.3 & 94.7 \\
\hline \multirow{2}{*}{ AUC } & $0.760(0.721-0.796)$ & $0.714(0.659-0.764)$ & $0.827(0.770-0.874)$ \\
\hline & $P<0.001$ & $P<0.001^{\dagger}$ & $P<0.001$ \\
\hline \multicolumn{4}{|l|}{ WHtR } \\
\hline WHtR cut-off & $>0.54$ & $>0.55$ & $>0.49$ \\
\hline Sensitivity (\%) & 61.8 & 60.5 & 80.0 \\
\hline Specificity (\%) & 89.5 & 90.0 & 80.0 \\
\hline \multirow{2}{*}{ AUC } & $0.794(0.756-0.828)$ & $0.767(0.715-0.814)$ & $0.834(0.778-0.881)$ \\
\hline & $P<0.001$ & $P<0.001$ & $P<0.001$ \\
\hline
\end{tabular}

AUC: area under the curve; 95\% CI in parentheses; ${ }^{\ddagger}$ AUC significantly different from WC $(P<0.05) ;{ }^{\dagger}$ AUC significantly different from WHtR $(P<0.05)$.

study. Further studies are needed to confirm or contrast our findings.

\section{Conclusion}

In conclusion, BMI, WC, and WHtR are all predictors of high MRS. Despite the small differences in the discriminatory capabilities among measures of adiposity, making it difficult to recommend the best measure of obesity, BMI seems to have the best trade-off between sensitivity and specificity to screen for high MRS in both sexes.

\section{Conflict of Interests}

All the authors declare not to have any conflict of interests.

\section{Acknowledgments}

This study was supported by FCT-MCTES Grants nos. BD/44422/2008, BPD/65180/2009, BSAB/1025/2010, and PTDC/DES/098309/2008 and by Azorean Government.

\section{References}

[1] D. S. Freedman, L. K. Khan, W. H. Dietz, S. R. Srinivasan, and G. S. Berenson, "Relationship of childhood obesity to coronary heart disease risk factors in adulthood: the Bogalusa heart study," Pediatrics, vol. 108, no. 3, pp. 712-718, 2001.

[2] R. Kelishadi, "Childhood overweight, obesity, and the metabolic syndrome in developing countries," Epidemiologic Reviews, vol. 29, no. 1, pp. 62-76, 2007.

[3] S. Cook, P. Auinger, C. Li, and E. S. Ford, "Metabolic syndrome rates in united states adolescents, from the national health and nutrition examination survey, 1999-2002," Journal of Pediatrics, vol. 152, no. 2, pp. 165-170.e2, 2008.

[4] J. M. Saland, "Update on the metabolic syndrome in children," Current Opinion in Pediatrics, vol. 19, no. 2, pp. 183-191, 2007.

[5] M. Hara, E. Saitou, F. Iwata, T. Okada, and K. Harada, "Waist-to-height ratio is the best predictor of cardiovascular disease risk factors in Japanese schoolchildren," Journal of atherosclerosis and thrombosis, vol. 9, no. 3, pp. 127-132, 2002.

[6] R. P. Gelber, J. M. Gaziano, E. J. Orav, J. E. Manson, J. E. Buring, and T. Kurth, "Measures of obesity and cardiovascular risk among men and women," Journal of the American College of Cardiology, vol. 52, no. 8, pp. 605-615, 2008.

[7] N. Mattsson, T. Rönnemaa, M. Juonala, J. S. A. Viikari, and O. T. Raitakari, "Childhood predictors of the metabolic syndrome in adulthood. The Cardiovascular Risk in Young Finns Study," Annals of Medicine, vol. 40, no. 7, pp. 542-552, 2008.

[8] C. Moreira, R. Santos, S. Vale et al., "Metabolic syndrome and physical fitness in a sample of Azorean adolescents," Metabolic Syndrome and Related Disorders, vol. 8, no. 5, pp. 443-449, 2010.

[9] T Lohman, A Roche, and F Martorell, Eds., Anthropometric Standardization Reference Manual, Human Kinetics, Champaign, Ill, USA, 1991.

[10] J. M. Tanner and R. H. Whitehouse, "Clinical longitudinal standards for height, weight, height velocity, weight velocity, and stages of puberty," Archives of Disease in Childhood, vol. 51, no. 3, pp. 170-179, 1976.

[11] W. P. Castelli, "Cholesterol and lipids in the risk of coronary artery disease. The Framingham Heart Study," Canadian Journal of Cardiology, vol. 4, pp. 5A-10A, 1988.

[12] D. R. Matthews, J. P. Hosker, and A. S. Rudenski, "Homeostasis model assessment: insulin resistance and $\beta$-cell function from fasting plasma glucose and insulin concentrations in man," Diabetologia, vol. 28, no. 7, pp. 412-419, 1985. 
[13] L. B. Andersen, M. Harro, L. B. Sardinha et al., "Physical activity and clustered cardiovascular risk in children: a crosssectional study (The European Youth Heart Study)," Lancet, vol. 368, no. 9532, pp. 299-304, 2006.

[14] C. Jung, N. Fischer, M. Fritzenwanger, and H. R. Figulla, "Anthropometric indices as predictors of the metabolic syndrome and its components in adolescents," Pediatrics International, vol. 52, no. 3, pp. 402-409, 2010.

[15] WHO, "Obesity: Preventing and managing the global epidemic: report of a WHO consultation," World Health Organization - Technical Report Series, pp. 1-253, 2000.

[16] T. J. Cole, M. C. Bellizzi, K. M. Flegal, and W. H. Dietz, "Establishing a standard definition for child overweight and obesity worldwide: international survey," British Medical Journal, vol. 320, no. 7244, pp. 1240-1243, 2000.

[17] C. Maffeis, A. Pietrobelli, A. Grezzani, S. Provera, and L. Tatò, "Waist circumference and cardiovascular risk factors in prepubertal children," Obesity Research, vol. 9, no. 3, pp. 179187, 2001.

[18] A. Soto González, D. Bellido, M. M. Buño et al., "Predictors of the metabolic syndrome and correlation with computed axial tomography," Nutrition, vol. 23, no. 1, pp. 36-45, 2007.

[19] M. Ashwell, "Waist to height ratio and the Ashwell@ shape chart could predict the health risks of obesity in adults and children in all ethnic groups," Nutrition and Food Science, vol. 35, no. 5, pp. 359-364, 2005.

[20] S. Nambiar, I. Hughes, and P. S. Davies, "Developing waistto-height ratio cut-offs to define overweight and obesity in children and adolescents," Public Health Nutrition, vol. 13, no. 10, pp. 1566-1574, 2010.

[21] S. C. Savva, M. Tornaritis, M. E. Savva et al., "Waist circumference and waist-to-height ratio are better predictors of cardiovascular disease risk factors in children than body mass index," International Journal of Obesity, vol. 24, no. 11, pp. 1453-1458, 2000.

[22] D. S. Freedman, M. K. Serdula, S. R. Srinivasan, and G. S. Berenson, "Relation of circumferences and skinfold thicknesses to lipid and insulin concentrations in children and adolescents: the Bogalusa Heart Study," American Journal of Clinical Nutrition, vol. 69, no. 2, pp. 308-317, 1999. 


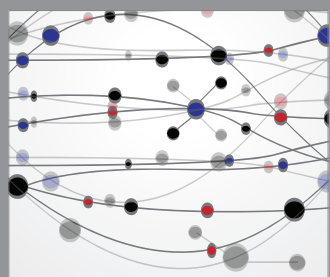

The Scientific World Journal
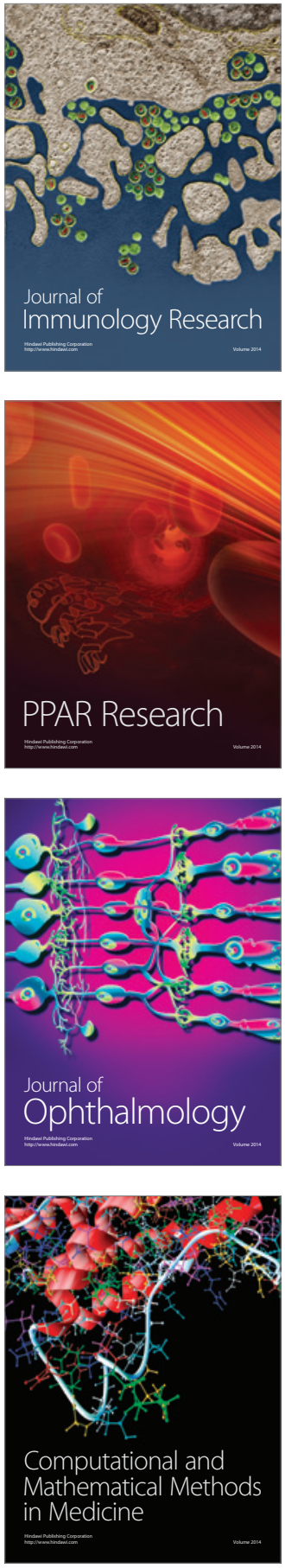

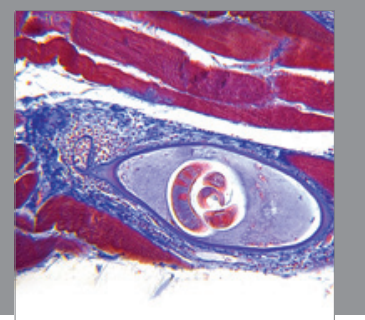

Gastroenterology

Research and Practice
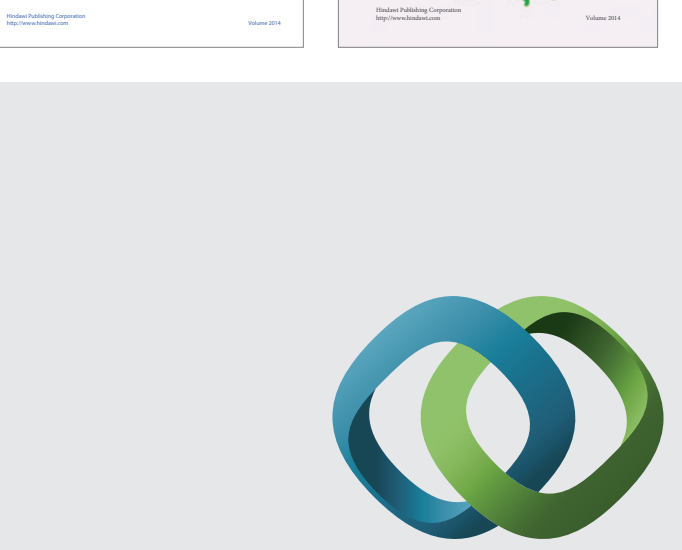

\section{Hindawi}

Submit your manuscripts at

http://www.hindawi.com
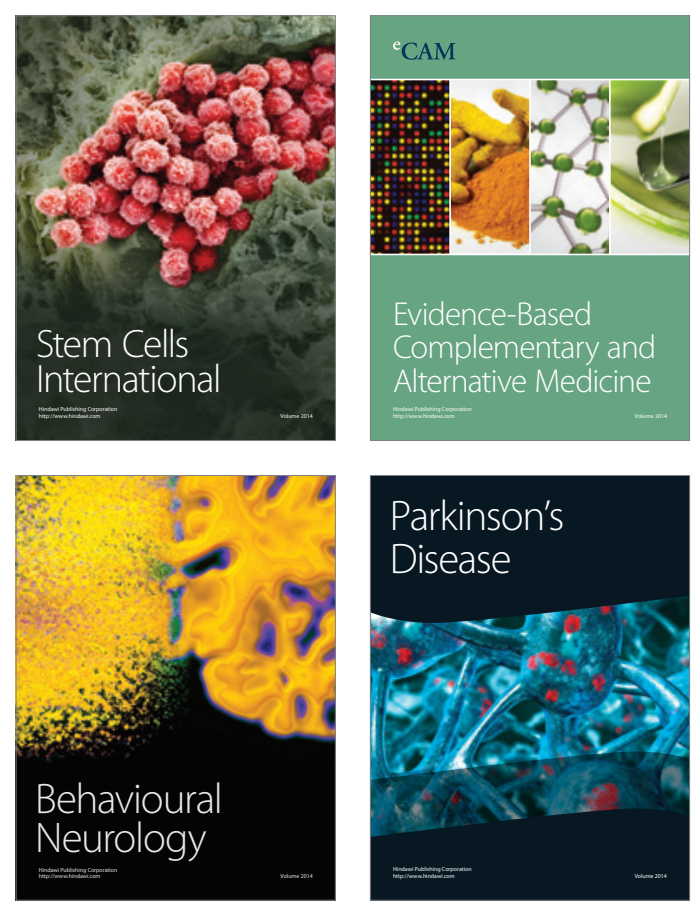

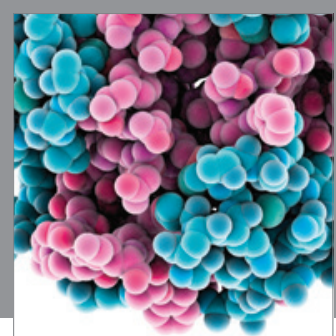

Journal of
Diabetes Research

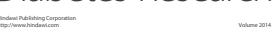

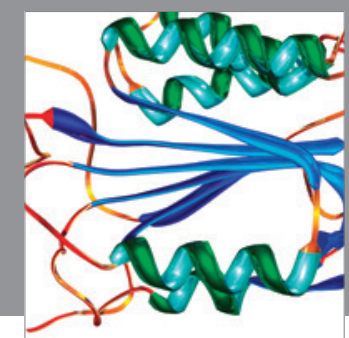

Disease Markers
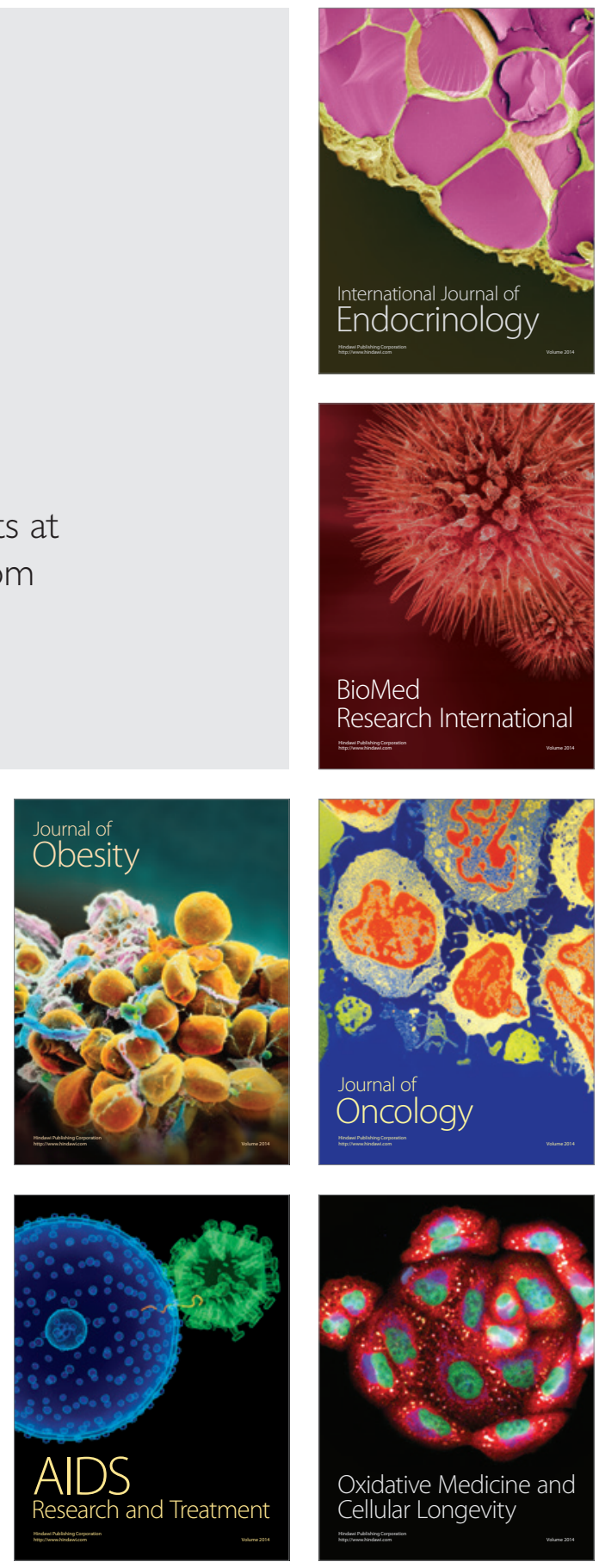